\title{
Effect of Iodide Ions on the Inhibitive Performance of O-, M-, P-Nitroaniline on Mild Steel in Hydrochloric Acid Solution
}

\section{1,20JO, FK; ${ }^{1}$ ADEJORO, IA ; ${ }^{* 3}$ AKPOMIE, KG; ${ }^{1,4}$ OGUNYEMI, BT; ${ }^{3}$ OYEKA, EE}

\author{
${ }^{I}$ Department of Chemistry (Physical Unit), University of Ibadan, Ibadan, Nigeria \\ ${ }^{2}$ Department of Chemical Sciences, Bingham University, Karu, Nigeria \\ ${ }^{3}$ Department of Pure \& Industrial Chemistry, University of Nigeria, Nsukka, Nigeria \\ ${ }^{4}$ Department of Chemistry, Federal University Otuoke, Bayelsa, Nigeria \\ *Corresponding Author Email: kovo.akpomie@unn.edu.ng
}

\begin{abstract}
The effect of iodide ions on the inhibitive performance of ortho, meta and para nitro aniline in $1 \mathrm{M} \mathrm{HCl}$ for mild steel has been studied using weight loss method (gravimetric) measurements at 303 and $333 \mathrm{~K}$. Results obtained show that the presence of the nitro aniline compounds in $1 \mathrm{M} \mathrm{HCl}$ solution inhibits the corrosion process of mild steel. Its adsorption was found to be physical, exothermic and spontaneous as confirmed by values of activation energy and free energy of adsorption (not up to $-20 \mathrm{~kJ} \mathrm{~mol}^{-1}$ for free energy of adsorption and below $80 \mathrm{~kJ} \mathrm{~mol}^{-1}$ for activation energy) and also fitted the Langmuir adsorption model. Addition of iodide ions synergistically increased the inhibition efficiency of the nitro aniline compounds. Quantitative Structure Activity Relationship (QSAR) approach was used on a composite index of some quantum chemical parameters. The results showed that the Inhibition Efficiency was closely related to some of the quantum chemical parameters.
\end{abstract}

\section{DOI: https://dx.doi.org/10.4314/jasem.v22i5.32}

Copyright: Copyright () 2018 Ojo et al. This is an open access article distributed under the Creative Commons Attribution License (CCL), which permits unrestricted use, distribution, and reproduction in any medium, provided the original work is properly cited.

Dates: Received: 12 April 2018; Revised: 22 April: 2018; Accepted: 26 April 2018

Keywords: Corrosion, Nitroaniline, Iodide ions, Theoretical study.

There has been a growing interest in the use of organic compounds as inhibitors for the aqueous corrosion of metals. The protection of metal surfaces against corrosion is an important industrial and scientific topic. The use of Inhibitor is one of the practical means of preventing corrosion (Adejoro et al., 2015). In acidic media inhibitors can adhere to a metal surface to form a protective barrier against corrosive agent in contact with metal. The effectiveness of inhibitors to provide corrosion protection depends to a large extent on the interaction between the inhibitor and the metal surface (Ameena, 2014). The adsorbed inhibitors can affect the corrosion reaction, either by the blocking effect of the adsorbed inhibitor on the metal surface or by the effect attributed to the change in the activation barriers of the anodic and cathodic reactions of the corrosion process (Eno et al., 2010).

Organic compounds, which can donate electrons to unoccupied $d$ orbital of metal surface to form coordinate covalent bonds, can also accept free electrons from the metal surface by using their anti bonding orbital to form feedback bonds, thus constituting excellent corrosion inhibitors. The most effective organic inhibitors are those compounds containing hetero atoms like nitrogen, oxygen, sulfur and phosphorus (Barouni et al., 2014). The presence of aromatic rings and conjugated double bonds could further enhance the effectiveness of these organic compounds as excellent corrosion inhibitors. The inhibitory activity of these molecules is accompanied by their adsorption to the metal surface. The free electron pairs on heteroatom or $\pi$ electrons are readily available for sharing to form bonds and act as nucleophile centers of inhibitors molecules and greatly facilitate the adsorption process over the metal surface, whose atoms acts as electrophiles.

Recently, the effectiveness of an inhibitor molecule has been related to its spatial as well as its electronic structure. Quantum chemical methods are ideal tools for investigating these parameters and can provide insight into the inhibitor - surface interaction (Musa and Kamal, 2015). The use of orto, meta and para nitro aniline as effective organic inhibitors is interesting and can offer a great deal of solution to the unending corrosion challenge. However, it has not being extensively reported as such few reports exist in literature to date. Thus the aim of this study is to investigate the efficiency of this nitro aniline in combination with potassium iodide with the view of 
enhancing the inhibition efficiency of these nitro aniline compounds.

\section{MATERIALS AND METHODS}

Materials:The steel metal sheets were obtained from building and material market, Maraba, Karu, Nasarawa state. Test were performed on a freshly prepared cylindrical rods of mild steel of the following composition (wt. \%): $0.012 \% \mathrm{Ni}, 0.017 \% \mathrm{Mo}$, $0.040 \% \mathrm{Cr}, 0.168 \% \mathrm{C}, 0.324 \% \mathrm{Mn}, 0.007 \% \mathrm{Si}$, $0.008 \% \mathrm{P}$ and balance Fe. Specimen used in the weight loss technique was mechanically press-cut into cylindrical rods, each of dimension $30 \mathrm{~mm}$ length and $1.45 \mathrm{~mm}$ diameter. The rods were degreased by washing in absolute ethanol, dried in acetone and preserved in a desiccator. Subsequently, the initial weight of each coupon was carefully measured with an Ohahus Pioneer ${ }^{\mathrm{TM}}$ analytical weighing balance.

Weight loss method (Gravimetric method):In the gravimetric experiment, a previously weighed metal (mild steel) coupon was completely immersed in 50 $\mathrm{mL}$ of the test solution in an open beaker. The beaker was inserted into a water bath maintained at $30{ }^{\circ} \mathrm{C}$ for $8 \mathrm{hr}$. The corrosion product was removed by washing each coupon (withdrawn from the test solution) in a solution containing $50 \% \mathrm{NaOH}$ and $100 \mathrm{~g}$ of zinc dust(Adejoro et al., 2015). The washed coupon was rinsed in acetone and dried in the air before reweighing. The experiment was also repeated at 40,50 and $60{ }^{\circ} \mathrm{C}$. In each case, the difference in weight at intervals of $2 \mathrm{hr}$ for a period of $8 \mathrm{hr}$ was taken as the total weight loss. From the weight loss results, the inhibition efficiency (I.E\%) of the inhibitor, degree of surface coverage and corrosion rates were calculated using equations 1,2 and 3 respectively.

$$
\begin{aligned}
& \text { \%I. E }=\left(1-\frac{W_{1}}{W_{2}}\right) 100 \\
& \Theta=1-\frac{W_{1}}{W_{2}}
\end{aligned}
$$

Where $W_{1}$ and $W_{2}$ are the weight losses (g) for mild steel in the presence and absence of the inhibitor solution, $\Theta$ is the degree of surface coverage of the inhibitor, $A$ is the total surface area of the mild steel $\operatorname{rod}\left(\mathrm{cm}^{2}\right), t$ is the period of immersion (hr) and $W$ is the weight loss of mild steel after time $(t)$. All the measurements were performed in triplicate and the mean value recorded.

Scanning electron microscopy (SEM):The analysis of the morphology of the mild steel surface after $72 \mathrm{hr}$ of immersion time was carried out using scanning electron microscopy (SEM), operated in the contact mode under ambient conditions using JSM-6010LA analytical scanning electron microscope. Images of the specimens were recorded after exposure as follows: (a) mild steel in the absence of inhibitor and acid, (b) mild steel in the presence of acid only and (c) mild steel in the presence of acid and inhibitors of concentration 10 x $10^{-3} \mathrm{M}$.

Quantum measurements: All the quantum chemical calculations were performed with SPARTAN'14 V1.0. The full optimization was initially achieved by using molecular mechanics force fields (MMFF). The results from MMFF were further selected as input and re-optimized using Semi empirical AM1. The Semi empirical AM1 structures were selected as input and were re-optimized using Density Functional Theory (DFT) at the level of B3LYP methods which uses the exchange functional proposal by Becke and all the correlations functional given by Lee, Yang and Parr. The b-31G* basis set has been used in conjunction with DFT method because it has the advantage of being flexible enough to guarantee reliable theoretical results and being small enough for rapid calculations. It represents an excellent compromise between completeness and economy. The molecular geometry was fully optimized without any constraint using analytical gradient procedure implemented within the program package. The following quantum chemical indices were considered: the energy of the highest occupied molecular orbital (Еном), the energy of the lowest unoccupied molecular orbital ( $\mathrm{E}_{\mathrm{LUMO}}$ ), separation energy $\left(\mathrm{E}_{\mathrm{LUMO}}-\mathrm{E}_{\text {Hомо }}\right.$ ), dipole moment $(\mu), \log P$, and Polarizability of the nitro aniline compounds.

\section{RESULT AND DISCUSSION}

Weight loss measurements: The weight loss method of monitoring corrosion rate is useful because of its simple application and reliability(Obot et al., 2009). Weight loss of mild steel, in mg, was determined at various time intervals in the absence and presence of different concentrations of the inhibitors studied. Figures 1 - 3 show the weight loss-time curves obtained for mild steel in $1 \mathrm{M} \mathrm{HCl}$ in the absence and presence of different concentrations of nitro aniline compounds at 303 and $333 \mathrm{~K}$. The figures show that the presence of the inhibitors falls significantly below that of the free acid. The calculated values of corrosion rates (CR) and inhibition efficiency (\%I.E) obtained from weight loss measurements for different concentrations of nitro aniline compounds in $1 \mathrm{M} \mathrm{HCl}$ after 8 hours immersion at 303 and $333 \mathrm{~K}$ are listed in Tables 1 and 2 respectively. It is evident from these tables and figures that the corrosion rate decreased with increasing inhibitor concentration but increased 
with rise in temperature. Table 2 also show that the inhibition efficiency (\% I.E) increased with increasing inhibitor concentration, reaching a maximum of 52:24 for ortho nitro aniline, 51:37 for meta nitro aniline and $50: 50 \%$ for para nitro aniline. This may be due to the adsorption of these compounds onto the mild steel surface through non-bonding electron pairs of nitrogen and oxygen atoms as well as the $\pi$-electrons of the aromatic rings. Similar observation has been reported (Karthik and Sundaravadivelu, 2016).

Adsorption Isotherm: Adsorption isotherm study describes the adsorptive behavior of organic inhibitors to know the adsorption mechanism (Dawodu and Akpomie, 2014). It depends mainly on the nature and charge of the metal surface, adsorption of solvent and other ionic species, electronic characteristics of the metal surface, temperature of the corrosion reaction and the electrochemical potential at solution interface. The description of adsorption processes can be achieved via the adoption of adsorption isotherms.

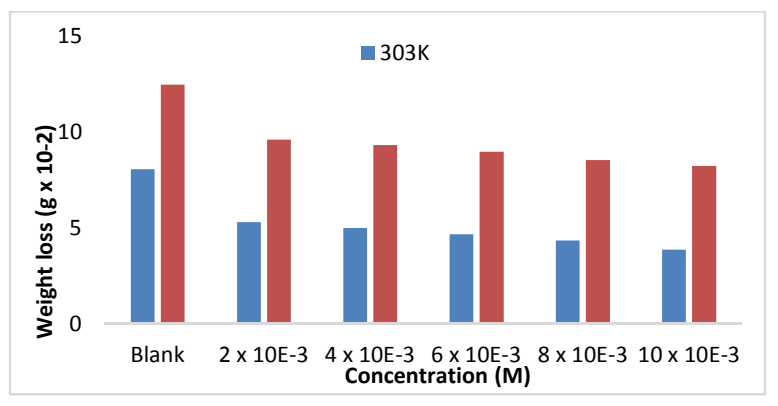

Fig1: Weight loss values against different concentrations of ortho nitro aniline in $1 \mathrm{M} \mathrm{HCl}$ of mild steel corrosion after 8hours immersion time at $303 \mathrm{~K}$ and $333 \mathrm{~K}$

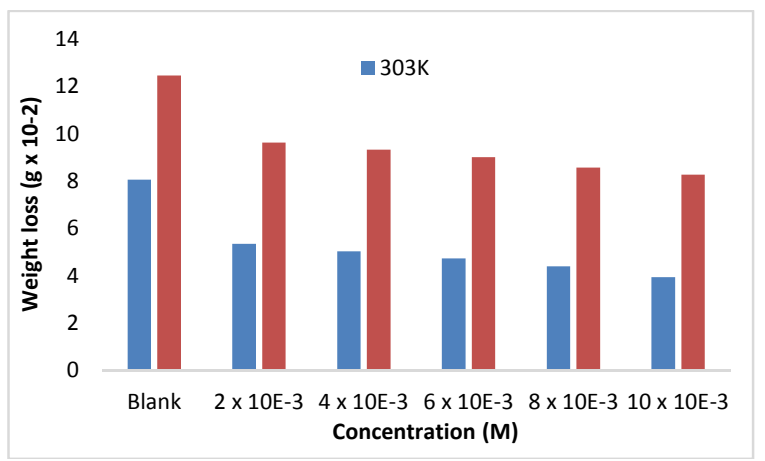

Fig2: Weight loss values against different concentrations of meta nitro aniline in $1 \mathrm{M} \mathrm{HCl}$ of mild steel corrosion after 8hours immersion time at $303 \mathrm{~K}$ and $333 \mathrm{~K}$

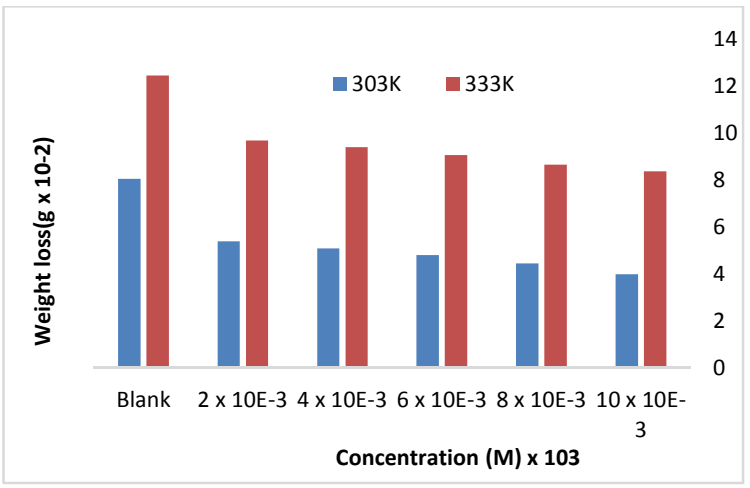

Fig 3: Weight loss values against different concentrations of para nitro aniline in $1 \mathrm{M} \mathrm{HCl}$ of mild steel corrosion after 8hours immersion time at $303 \mathrm{~K}$ and $333 \mathrm{~K}$

Table 1: Corrosion rates (CR) for mild steel in $1 \mathrm{M} \mathrm{HCl}$ in the presence and absence of different concentrations of nitro aniline compounds at temperatures of $303 \mathrm{~K}$ and $333 \mathrm{~K}$ using weight loss technique.

\begin{tabular}{lllllll}
\hline Concentration & \multicolumn{2}{l}{$\begin{array}{l}\text { Ortho nitro aniline } \\
\text { Corrosion rate }\left(\mathrm{mg} \mathrm{cm}^{-2} \mathrm{~h}^{-1}\right)\end{array}$} & \multicolumn{2}{c}{$\begin{array}{l}\text { Ceta nitro aniline } \\
\text { Corrosion rate }\left(\mathrm{mg} \mathrm{cm}^{-2} \mathrm{~h}^{-1}\right)\end{array}$} & \multicolumn{2}{l}{$\begin{array}{l}\text { Para nitro aniline } \\
\text { Corrosion rate }\left(\mathrm{m} \mathrm{cm}^{-2} \mathrm{~h}^{-1}\right)\end{array}$} \\
& $303 \mathrm{~K}$ & $333 \mathrm{~K}$ & $303 \mathrm{~K}$ & $333 \mathrm{~K}$ & $303 \mathrm{~K}$ & $333 \mathrm{~K}$ \\
\hline Blank & 7.18 & 11.11 & 7.18 & 11.11 & 4.18 & 11.11 \\
$2 \times 10^{-3} \mathrm{M}$ & 4.71 & 8.54 & 4.75 & 8.57 & 4.81 & 8.64 \\
$4 \times 10^{-3} \mathrm{M}$ & 4.44 & 8.23 & 4.47 & 8.30 & 4.54 & 8.38 \\
$6 \times 10^{-3} \mathrm{M}$ & 4.15 & 7.99 & 4.21 & 8.02 & 4.29 & 8.08 \\
$8 \times 10^{-3} \mathrm{M}$ & 3.86 & 7.60 & 3.91 & 7.63 & 3.97 & 7.71 \\
$10 \times 10^{-3} \mathrm{M}$ & 3.43 & 7.32 & 3.49 & 7.36 & 3.55 & 7.47 \\
\hline
\end{tabular}

Table 2: Inhibition Efficiency (IE\%) for mild steel in $1 \mathrm{M} \mathrm{HCl}$ in the presence and absence of different concentrations of nitro aniline

\begin{tabular}{|c|c|c|c|c|c|c|}
\hline \multirow[t]{2}{*}{ Concentration } & \multicolumn{2}{|c|}{$\begin{array}{l}\text { Ortho nitro aniline } \\
\text { Inhibition Efficiency (\%) }\end{array}$} & \multicolumn{2}{|c|}{$\begin{array}{l}\text { Meta nitro aniline } \\
\text { Inhibition Efficiency (\%) }\end{array}$} & \multicolumn{2}{|c|}{$\begin{array}{l}\text { Para nitro aniline } \\
\text { Inhibition Efficiency }(\%)\end{array}$} \\
\hline & $303 K$ & $333 K$ & $303 \mathrm{~K}$ & $333 \mathrm{~K}$ & $303 \mathrm{~K}$ & $333 \mathrm{~K}$ \\
\hline Blank & & & & & & \\
\hline $2 \times 10^{-3} \mathrm{M}$ & 34.45 & 23.07 & 33.83 & 22.83 & 32.96 & 22.19 \\
\hline $4 \times 10^{-3} \mathrm{M}$ & 38.18 & 25.40 & 37.69 & 25.24 & 36.82 & 24.60 \\
\hline $6 \times 10^{-3} \mathrm{M}$ & 42.16 & 28.05 & 41.42 & 27.81 & 40.30 & 27.25 \\
\hline $8 \times 10^{-3} \mathrm{M}$ & 46.27 & 31.59 & 45.52 & 31.27 & 44.65 & 30.55 \\
\hline $10 \times 10^{-3} \mathrm{M}$ & 52.24 & 34.08 & 51.37 & 33.76 & 50.50 & 32.72 \\
\hline
\end{tabular}

The most frequently used isotherms include; Langmuir, Frumkin, Hill de Boer, Flory-Huggins, Parsons-Temkin, Dhar-Flory-Huggins, and Bockris-
Swinkels. The establishment of adsorption isotherms that describe the adsorption of a corrosion inhibitor can provide important clues to the nature of the metal- 
inhibitor interaction. Adsorption of the organic molecules occurs as the interaction energy between molecule and metal surface is higher than that between the water molecule and the metal surface.In order to obtain the adsorption isotherm, the degree of surface coverage $(\theta)$ for various concentrations of the inhibitor has been calculated using equation 4. Langmuir isotherm was tested for its fit to the experimental data. Langmuir isotherm is given by:

$C / \theta=1 / K_{a d s}+C$

Where $\theta$ is the degree of surface coverage, $\mathrm{C}$ the molar inhibitor concentration in the bulk solution and $\mathrm{K}_{\mathrm{ads}}$ is the equilibrium constant of the process of adsorption. The plot of $\mathrm{C} / \theta$ versus $\mathrm{C}$ was linear according to Figures 4-6 with a correlation greater than 0.9 and slope deviated from unity (Table 3 ). The deviation of the slope from unity suggest that there is more than one active site having influence on the adsorption per molecule which signifies adherence of ortho, meta and para nitro aniline to the adsorption of the Langmuir model.

The values of the adsorption equilibrium constant $\left(\mathrm{K}_{\mathrm{ads}}\right)$ obtained from the intercept of the Langmuir adsorption isotherms are related to the free energy of adsorption according to equation 5

$\Delta G^{o}$ ads $=-2.303 R T \log \left(55.5 K_{a d s}\right) 5$

Where $\Delta \mathrm{G}^{\mathrm{o}}$ ads is the free energy of the adsorption, $\mathrm{R}$ is the gas constant and $\mathrm{T}$ is the temperature of the system. Calculated values of the free energies are also presented in Table 3. The free energies ranged from 7.0773 to $-6.7200 \mathrm{~kJ} / \mathrm{mol}$ and are within the range expected for the transfer of charge from the inhibitor to the metal surface. Thus, the adsorption of ortho, meta and para nitro aniline were spontaneous.

Generally, values of $\Delta \mathrm{G}^{\mathrm{o}}$ ads up to $-20 \mathrm{kJmol}^{-1}$ signify physiosorption (Dawodu and Akpomie, 2014), the inhibition acts due to electrostatic interaction between the charge molecules and the charged metal, while values around $-40 \mathrm{kJmol}^{-1}$ or more are associated with chemisorption as a result of sharing or transfer of electrons from the organic molecules to the metal surface to form a coordinate type of bond (Chemisorption) (Eno et al., 2010). The values obtained from this research ranged from -7.0773 to $6.7200 \mathrm{KJ} / \mathrm{mol}$, which supported the mechanism of physical adsorption

Thermodynamic considerations: The relationship between the percentage inhibition efficiency (IE\%) of an inhibitor, temperature and the activation energy of in the presence of an inhibitor was given (Ibeji et al., 2015) (a) Inhibitor whose IE (\%) decreases with increase in temperature.

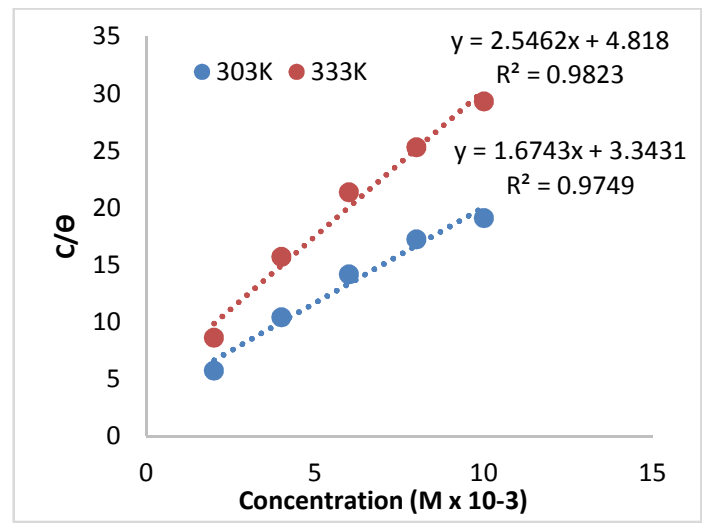

Fig 4: Langmuir isotherm for the adsorption of ortho nitro aniline on mild steel

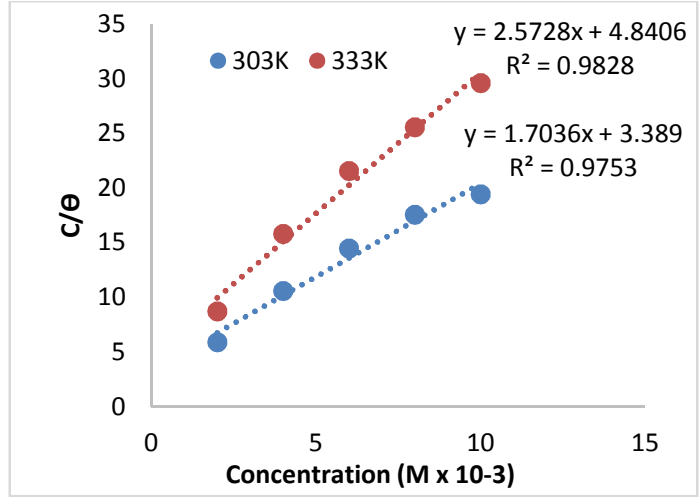

Fig 5: Langmuir isotherm for the adsorption of meta nitro aniline on mild steel in $1 \mathrm{M} \mathrm{HCl}$

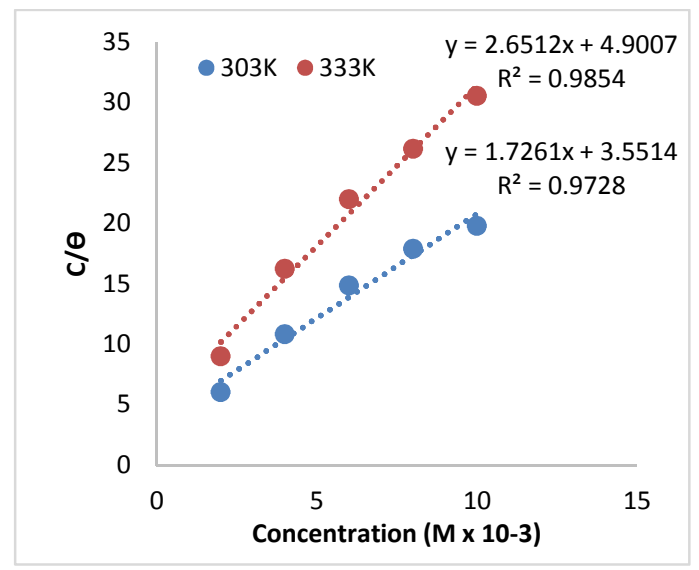

Fig 6: Langmuir isotherm for the adsorption of para nitro aniline on mild steel in $1 \mathrm{M} \mathrm{HCl}$

The value of activation energy ( $\mathrm{E}_{\mathrm{a}}$ ) found is greater than that in the uninhibited solution. (b) Inhibitors with IE (\%) that does not change with variation in temperature (i.e. either an increase or decrease). The activation energy $\left(E_{a}\right)$ does not change in the presence or absence of inhibitors. (c) Inhibitors whose IE (\%) 
tend to increase with an increase in temperature. The value of activation energy $\left(E_{a}\right)$ found is less than that of the uninhibited solution. A higher value of the activation energy $\left(E_{a}\right)$ of the process in an inhibitor's presence when compared to that in its absence can be attributed to physiosorption, while the opposite case can be attributed to chemisorptions.

To further show that the nitro aniline compounds adsorbed to mild steel via physiosorption, the values of activation energy $\left(E_{a}\right)$ were calculated with the help of Arrhenius equation:

$$
\log \left(C R_{2} / C R_{I}\right)=\mathrm{E}_{\mathrm{a}} / 2.303 \mathrm{R}\left(1 / \mathrm{T}_{1}-1 / \mathrm{T}_{2}\right) \quad 6
$$

Where $C R_{1}$ and $C R_{2}$ represent the corrosion rates at temperature $T_{1}$ and $T_{2}$, respectively. The values are as shown in Table 4. An increase in the value of $E_{a}$ in the presence of the nitro aniline compounds in comparison to that in its absence, and the decrease of its IE\% with temperature increase can be interpreted as an indication of physical adsorption. Also, it is expected that for chemical adsorption, the values of activation energy should be greater than $80 \mathrm{kJmol}^{-1}$ for the mechanism of physical adsorption (Abdullatef, 2015). With the range of $E_{a}$ values observed in Table 4 $\left(16.38-21.20 \mathrm{kJmol}^{-1}\right)$, it further confirms physical adsorption. An estimate of heat of adsorption was also obtained for the trend of surface coverage with temperature as follows:

$\mathrm{Q}_{\mathrm{ads}}=2.303 \mathrm{R}\left[\log \left(\theta_{2} / 1-\theta_{2}\right)-\log \left(\theta_{1} / 1-\theta_{1}\right)\right] \times\left(\mathrm{T}_{1} \times \mathrm{T}_{2} / \mathrm{T}_{2}-\mathrm{T}_{1}\right) \quad 7$

Where $\theta_{1}$ and $\theta_{2}$ are the degrees of surface coverage and the temperatures $T_{1}$ and $T_{2}$. The calculated values for $\mathrm{Q}_{\mathrm{ads}}$ are presented in Tables 4. Values of $\mathrm{Q}_{\mathrm{ads}}$ calculated via equation 7 ranged from -20.67 to -14.59 $\mathrm{kJmol}^{-1}$. These values are negative indicating that the adsorption of these nitro aniline compoundson mild steel is exothermic (Abdullatef, 2015).

Table 3: Langmuir parameters for the adsorption of 3 methyl indole on mild steel surface

\begin{tabular}{lllllll}
\hline Temperature & Molecule & Kads & $\Delta \mathrm{Gads}$ & Slope & $\mathrm{R}^{2}$ & Intercept \\
\hline $303 \mathrm{~K}$ & Ortho nitro aniline & 0.2991 & 7.0773 & 1.6743 & 0.9749 & 3.3431 \\
& Meta nitro aniline & 0.2951 & 7.0434 & 1.7063 & 0.9753 & 3.389 \\
& Para nitro aniline & 0.2816 & 6.9254 & 1.7261 & 0.9728 & 3.5514 \\
$333 \mathrm{~K}$ & Ortho nitro aniline & 0.2076 & 6.7670 & 2.5462 & 0.9823 & 4.818 \\
& Meta nitro aniline & 0.2066 & 6.7537 & 2.5728 & 0.9828 & 4.8406 \\
& Para nitro aniline & 0.2041 & 6.7200 & 2.6512 & 0.9854 & 4.9007 \\
\hline
\end{tabular}

Table 4: Thermodynamic parameters for the adsorption of ortho, meta and para nitro aniline

\begin{tabular}{lllllll}
\hline $\begin{array}{l}\text { Concentration } \\
\mathrm{mol} / \mathrm{dm}^{3}\end{array}$ & \multicolumn{2}{l}{ Ortho nitro aniline } & \multicolumn{2}{l}{ Meta nitro aniline } & \multicolumn{2}{l}{ Para nitro aniline } \\
& $\begin{array}{l}\text { Activation } \\
\text { energy, Ea } \\
(\mathrm{KJ} / \mathrm{mol})\end{array}$ & $\begin{array}{l}\text { Heat of } \\
\text { adsorption, } \\
\mathrm{Q}_{\text {ads }}(\mathrm{KJ} / \mathrm{mol})\end{array}$ & $\begin{array}{l}\text { Activation } \\
\text { energy, Ea } \\
(\mathrm{KJ} / \mathrm{mol})\end{array}$ & $\begin{array}{l}\text { Heat of } \\
\text { adsorption, } \\
\mathrm{Q}_{\text {ads }}(\mathrm{KJ} / \mathrm{mol})\end{array}$ & $\begin{array}{l}\text { Activation } \\
\text { energy, Ea } \\
(\mathrm{KJ} / \mathrm{mol})\end{array}$ & $\begin{array}{l}\text { Heat of } \\
\text { adsorption, } \\
\mathrm{Q}_{\text {ads }}(\mathrm{KJ} / \mathrm{mol})\end{array}$ \\
\hline Blank & 12.21 & - & 12.21 & - & 12.21 & - \\
$2 \times 10^{-3}$ & 16.64 & -15.27 & 16.50 & -14.89 & 16.38 & -14.59 \\
$4 \times 10^{-3}$ & 17.26 & -16.73 & 17.31 & -16.42 & 16.97 & -16.18 \\
$6 \times 10^{-3}$ & 18.32 & -17.91 & 18.02 & -17.45 & 17.71 & -16.96 \\
$8 \times 10^{-3}$ & 18.95 & -17.93 & 18.70 & -17.60 & 18.56 & -17.59 \\
$10 \times 10^{-3}$ & 21.20 & -20.64 & 20.87 & -20.37 & 20.81 & -20.67 \\
\hline
\end{tabular}

Effect of iodide ion addition: Halides have been reported to inhibit the corrosion of some metals in strong acids and this effect depends on the ionic size and charge, the electrostatic field set up by the negative charge of the anion on the adsorption sites and the nature and concentration of the halide ion (Bouraadaet al., 2016). The inhibitive effect increases in the order $\mathrm{Cl}-<\mathrm{Br}-<\mathrm{I}-$, which seems to indicate that the radii of halide ions may have an important role to play. For example, the iodide ion (radius: $135 \mathrm{pm}$ ) is more predisposed to adsorption than is the bromide ion (radius: 114 pm) or chloride ion (radius: 90 pm). From Table 5, it is seen that the inhibition efficiency of nitro aniline compounds are further enhanced by the addition of $1 \times 10^{-4} \mathrm{M}$ KI. The inhibition efficiency significantly increased to $73.63 \%$ for ortho nitro aniline, $72.64 \%$ for meta nitro aniline and $70.77 \%$ for para nitro aniline at $303 \mathrm{~K}$. This synergistic inhibitive effect brought about by the presence of indole derivatives in combination with potassium iodide (KI) on the corrosion of mild steel in $1 \mathrm{M} \mathrm{HCl}$ can be explained as follows: The strong adsorptions of iodide ions on the metal surface is responsible for the synergistic effect of iodide ion, in combination with the indole derivatives in their cationic forms respectively (Muralidharam et al., 1997). The inhibitors are then absorbed by columbic attraction on the metal surface where iodide ions are already adsorbed. Stabilization of adsorbed iodide ions with inhibitors in cationic forms lead to greater surface coverage and thereby greater inhibition.

The synergistic parameters were calculated using equation 8 :

$S_{p}=\frac{1-l_{1+2}}{1-l_{1+2}}$ 8 
Where $\mathrm{I}_{1+2}=\left(\mathrm{I}_{1}+\mathrm{I}_{2}\right) ; \mathrm{I}_{1}=$ inhibition efficiency of the halides, $\mathrm{I}_{2}=$ inhibition efficiency of the indole derivatives, $I^{\prime}=$ measured inhibition efficiency for the indole derivatives in combination with iodide ions. $S_{p}$ approaches 1 when no interaction between the inhibitor compounds exists, while $S_{p}>1$ indicates a synergistic effect. In the case of $S_{p}<1$, the antagonistic interaction prevails, which may be attributed to competitive adsorption. Values of $S_{p}$ for the indole derivatives in combination with potassium iodide (KI) are presented in Table 6 . The $S_{\mathrm{p}}$ values obtained are greater than one $\left(S_{p}>1\right)$, thereby suggesting that the improved inhibition efficiency caused by the addition of iodide ions to the indole derivatives is only due to synergistic effect. Thus it can be suggested that iodide ion $\left(\mathrm{I}^{-}\right)$is initially adsorbed on the metal surface. The nitro aniline compounds in the cationic form are then adsorbed by the columbic attraction on the metal surface, where the iodide ions are already adsorbed and thus suppress the self-corrosion rate by the stabilization of the adsorbed anion and by the increase in surface coverage. The organic cation itself is weakly adsorbed on the surface of the mild steel, since the metal surface is positively charged in $\mathrm{HCl}$ solution. However, adsorption of iodide ion caused a decrease in the positive charge of Fe due to the formation of Feanion surface bond which produces a negative pole enabling the adsorption of the organic cation.
Table 5: Inhibition efficiencies (I.E\%) for mild steel in $1 \mathrm{M} \mathrm{HCl}$ in the presence and absence of $10 \times 10^{-3} \mathrm{M}$ nitro aniline compounds

\begin{tabular}{ll}
\multicolumn{2}{c}{ and $1 \times 10^{-4} \mathrm{M}$ potassium iodide $(\mathrm{KI})$ at $303 \mathrm{~K}$} \\
\hline Molecule & Inhibition \\
& Efficiency $(\%)$ \\
\hline ortho nitro aniline & 52.24 \\
meta nitro aniline & 51.37 \\
para nitro aniline & 50.50 \\
KI $\quad 43.16$ \\
ortho nitro aniline + KI & 73.63 \\
\multicolumn{1}{c}{ meta nitro aniline + KI } & 72.64 \\
para nitro aniline + KI & 70.77 \\
\hline
\end{tabular}

Table 6: Synegistic parameters $\left(\mathrm{S}_{\mathrm{p}}\right)$ of $10 \times 10^{-3} \mathrm{M}$ nitro aniline compounds with $1 \times 10^{-4} \mathrm{M}$ potassium iodide in $1 \mathrm{M} \mathrm{HCl}$ at $303 \mathrm{~K}$.

\begin{tabular}{ll}
\hline Molecule & $\mathrm{S}_{\mathrm{p}}$ \\
\hline ortho nitro aniline & 2.31 \\
meta nitro aniline & 2.32 \\
para nitro aniline & 2.34 \\
\hline
\end{tabular}

Scanning electron microscopy: The scanning electron microscopy (SEM) image in Figure 8 clearly shows that the corrosion reaction does not take place homogeneously over the surface of mild steel in $1 \mathrm{M}$ $\mathrm{HCl}$ solution. However, the surface is remarkably protected by the nitro aniline compounds in comparison to the inhibitor - free solution. It has been established that the inhibition efficiency of these nitro aniline compounds are further enhanced by the addition of $1 \times 10^{-4} \mathrm{M} \mathrm{KI}$. This is clearly shown in Figure 8.
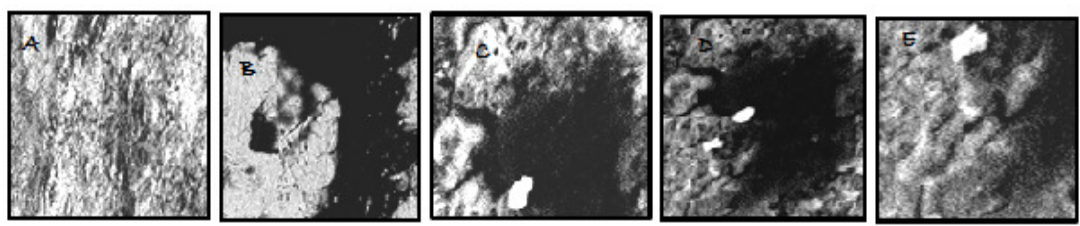

Fig 7: SEM characteristics of the mild steel in $1.0 \mathrm{M} \mathrm{H}_{2} \mathrm{SO}_{4}$ in (a) mild steel in the absence of inhibitor and acid, (b) mild steel in the presence of acid only (c) mild steel in the presence of ortho nitro aniline and acid, (d) mild steel in the presence of meta nitro aniline and acid (e) mild steel in the presence of para nitro aniline and acid
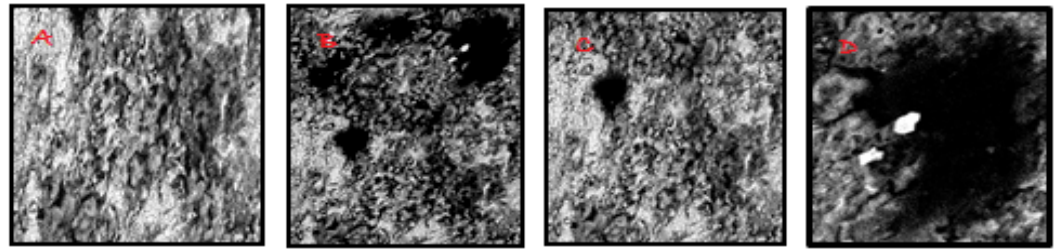

Fig 8: (a) mild steel in the presence of ortho nitro aniline, KI and acid, (b) mild steel in the presence of meta nitro aniline KI and acid, (c) mild steel in the presence of para nitro aniline, KI and acid (d) mild steel in the presence of KI and acid.

Quantum chemical study of orto, meta and para nitro aniline: The inhibition efficiency of the nitro aniline compounds on the corrosion of metal surface has been measured experimentally. Quantum chemical parameters obtained from the calculations which are responsible for the inhibition efficiency of inhibitors such as the energies of frontier molecular orbitals ( $\mathrm{E}_{\text {HOMO }}$ and $\mathrm{E}_{\mathrm{LUMO}}$ ), separation energy ( $\mathrm{E}_{\mathrm{LUMO}}-$

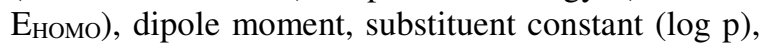

polarizability, molecular volumes and molecular weights are tabulated in Table 7.

It is obvious from the molecular structure (Figures 9 and 10) that the investigated nitro aniline compounds contain the amino group which has basic properties due to the presence of lone pair of electrons of the nitrogen atom. The importance of the basic properties of $\mathrm{NH}_{2}$ group is the formation of complexes of amine with metal ions. The $\mathrm{E}_{\text {Hомо }}$ is often associated with 
the electron donating ability of molecule (Arslan et al., 2009).

Table 7: Quantum chemical parameters of Nitro aniline derivatives using DFT method

\begin{tabular}{llll}
\hline Quantum chemical & Molecul & Molecule & Molecule \\
parameters & e 1 & 2 & 3 \\
\hline $\mathrm{E}_{\text {Homo }}(\mathrm{eV})$ & -6.06 & -6.14 & -6.25 \\
$\mathrm{E}_{\text {Lumo }}(\mathrm{eV})$ & -2.17 & -2.24 & -1.96 \\
$\mathrm{E}_{\text {Lumo }} \mathrm{E}_{\text {Homo }}(\mathrm{eV})$ & 3.89 & 3.90 & 4.29 \\
Dipole moment $(\mu)$ & 4.74 & 5.65 & 7.12 \\
Log P & -4.35 & -4.35 & -4.35 \\
Polarizability & 51.02 & 51.10 & 50.99 \\
Volume $\left(\AA^{3}\right)$ & 130.30 & 131.19 & 131.12 \\
Weight $(\mathrm{amu})$ & 138.126 & 138.126 & 138.126 \\
\hline
\end{tabular}

Therefore, increasing values of $\mathrm{E}_{\mathrm{HOMO}}$ indicates higher tendency for donation of electrons to the appropriate acceptor molecule with low energy and empty molecular orbital, thus increasing values of $\mathrm{E}_{\mathrm{HOMO}}$ facilitate the adsorption of the inhibitor (Eno et al., 2010). The E EUMO indicates the ability of the molecule to accept electrons. Hence, the lower the value of $\mathrm{E}_{\text {LUMO }}$ the more apparent for the molecule to accept electrons (Adejoro et al., 2015). The separation energy which indicates reactivity is the energy difference between the HOMO and the LUMO. The energy gap also relates to how soft or hard a molecule is. A larger energy gap indicates low reactivity to chemical specie, thus a soft molecule is more reactive than a hard molecule. The binding ability of the inhibitor to the metal surface increases with increasing the energy of HOMO, decreasing energy of LUMO and low separating energy values.

The values of $\log \mathrm{p}$ (substituent constant) were found to have a good relationship with the corrosion inhibition efficiencies of the studied inhibitors. Increasing the value of $\log \mathrm{p}$ increases the inhibition efficiencies of organic inhibitors. As the value of molecular volume and weight increase, so also the likely corrosion inhibition potentials of the molecules increase (Issa et al., 2009).The calculated quantum chemical parameters show that ortho nitro aniline with HOMO and the LUMO orbital energies at $-6.06 \mathrm{eV}$ and $-2.17 \mathrm{eV}$, respectively and separation energy of $3.89 \mathrm{eV}$ this clearly marks ortho nitro aniline a corrosion inhibitor with slightly higher reactivity toward the metal surface as compared to meta nitro aniline and para nitro aniline. The effect of nitro substituent on the meta and para position in the nitro aniline shows that the separation energy increases to $3.90 \mathrm{eV}$ for meta nitro aniline and $4.29 \mathrm{eV}$ for para nitro aniline. Similar quantum chemical values were calculated for $\log \mathrm{p}$, polarizability, molecular weight and molecular volume except for dipole moment. These results are consistent with experimental results. This great inhibiting potential could also be attributed to the aromatic ring having sufficient $\pi$ electrons thereby reducing the energy gap between the HOMO and LUMO orbitals. The calculated Mulliken charge shows that the nitrogen atom of the amine group and the oxygen atoms could act as active centers for the adsorption of the nitro aniline compounds molecule on the metal surface. The labeled optimized structure of nitro aniline compounds is shown in Figure 10 while the charge distribution over the whole molecule is shown in Figure 10.

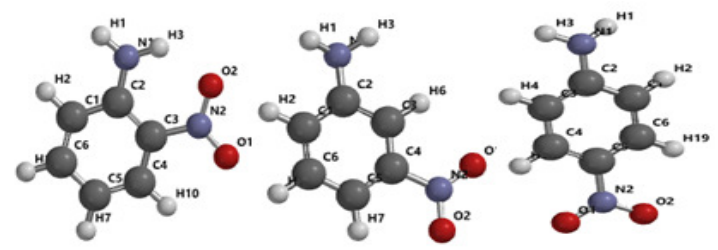

Fig 9: Labeled optimized structures of nitro aniline compounds
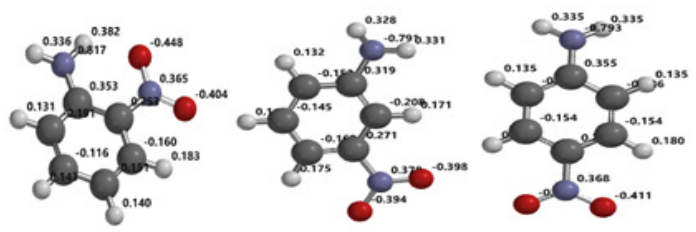

Fig 10: Optimized molecular structures of nitro aniline compounds showing mulliken charges.

Molecular orbital calculations are performed to determine the molecular orbital coefficients of the HOMO and LUMO levels for the studied nitro aniline compounds as corrosion inhibitors to explain the mechanism of their adsorptions on the metal surface. The HOMO electronic density distributions for the nitro aniline compounds are shown in Figure 12. It is evident from the calculations that the highest coefficients are found on the nitrogen atom of the amino group and the phenyl moiety which can be represented as active adsorption centres of the inhibitors. The adsorption of nitro aniline compounds occurs through the lone pairs of electrons of the nitrogen $\left(\mathrm{N}_{1}\right)$ atom, oxygen atoms and $\pi$ charges of the phenyl moiety. It is obvious that more than one adsorbed site could be obtained from one molecule of nitro aniline compounds. It was also pointed out by the Langmuir isotherm plots, which indicates a multicentre adsorption on the iron surface. The deviation of the slope from unity suggests that there is more than one active site having influence on the adsorption per molecule. This result is in positive agreement with quantum calculations (Obot et al., 2009). The LUMO plot is shown in Figure 12. The highest coefficient is localized on the nitrogen atom which can facilitate the back donation from the metal surface to the amino group of the inhibitor. This will increase its adsorption on the metal surface and accordingly increases its 
inhibition efficiency. The adsorption mechanism of the nitro aniline compounds on the metal surface can be explained as electron donation from the inhibitor molecule to the metal surface and back donation from the d-orbital of the metal surface to the $\pi$ anti-bonding counterpart of the inhibitor (Bouraada et al., 2016). In summary the above results indicate that the nitro aniline compounds are adsorbed on the metal surface through the nitro group, amine group and the highly electronegative nitrogen atom, the oxygen atoms and the $\pi$ charge of the phenyl moieties.
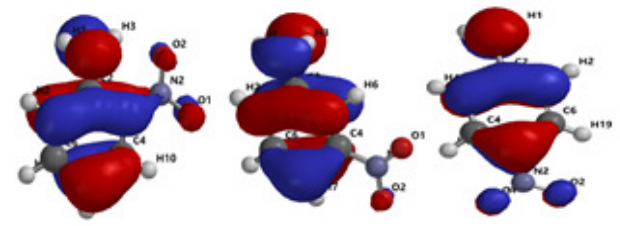

Fig 11: HOMO plot of optimized molecules of nitro aniline compounds

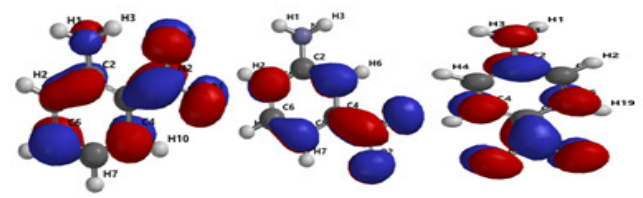

Fig 12: LUMO plot of optimized molecules of nitro aniline compounds

\section{Conclusions}

The presence of the nitro aniline compounds increased the corrosion activation energy and the heat of adsorption had negative values. Addition of iodide ions synergistically increased the inhibition efficiency of the nitro aniline compounds. Quantum chemical study reveals that the nitro aniline compounds are adsorbed on the metal surface through the nitro group, amine group and the highly electronegative nitrogen atom, the oxygen atoms and the $\pi$ charge of the phenyl moieties..The study showed the effectiveness of nitro aniline compounds in inhibiting the corrosive effect of mild steel in acid media.

\section{REFERENCES}

Abdullatef O.A. (2015). Kinetics and thermodynamics of the dissolution of steel in hydrochloric acid solution in the presence of silenemarmarica as environmentally friendly corrosion inhibitor, Egypt J. Petrol. 24: 505-511.

Adejoro I. A, Ojo F.K, Obafemi S.K. (2015). Corrosion inhibition potentials of ampicillin for mild steel in hydrochloric acid solution, J. Taibah Uni. Sci. 9:196-202.

Ameena M.A. (2014) Cefadroxil as save corrosion inhibitor for carbon steel in hydrochloric acid solutions. Inter. J. Adv. Res. 2: 522-534.
Arslan T, Kandemirli F, Ebenso E.E, Love I Alemu H. (2009). Quantum chemical studies on the corrosion inhibition of some sulphonamides on mild steel in acidic medium, Corros. Sci. 51:3547.

Barouni, K, Kassale A, Albourine A, Jbara O, Hammouti B, Bazzi I. (2014). Amino acids as corrosion inhibitors for copper in nitric acid medium: experimental and theoretical study. J. Mater. Environ.Sci. 5: 456-463.

Bouraada M, Quali M.S., Menorval L.C. (2016). Dodecylsulfate and dodecybenzenesulfonate intercalated hydrotalcites as adsorbent materials for the removal of BBR and from aqueous solution, $J$. Saudi. Chem. Soc. 20: 397-404.

Dawodu F.A, Akpomie K.G. (2014). Simultaneous adsorption of $\mathrm{Ni}$ (II) and $\mathrm{Mn}$ (II) ions from aqueous solution unto a Nigerian kaolinite clay, J. Mater. Res. Technol. 3,129-141.

Eno E, Ebenso E.E, Isabirye D.A, Eddy N.O. (2010). Potentials of Some Thiosemicarbazides for the Corrosion of Mild Steel in Acidic Medium, Int. J. Mol. Sci. 11: 2473-2498.

Ibeji C.U, Adejoro I.A, Adeleke B.B. (2015). A benchmark study on the properties of unsubstituted and some substituted polypyroles, J. Phys. Chem. Biophy. 5:3-11.

Issa R.M, Award M.K, Atlam F.M. (2009). DFT theoretical studies of antipyrines chiffbases as corrosion inhibitors. Mater. Corr. 60 (2009)1-6.

Karthik G.M, Sundaravadivelu S. (2016). Studies on the inhibition of mild steel corrosion in hydrochloric acid solution by atenotol drug. Egypt J. Petrol. 25:183-191.

Muralidharam S, Syed A.S, Berchmans I.J, Iyer S.V.K. (1997). Corrosion inhibition of mild steel in acid medium, Anti-Corrosion Mater. 44:30-39

Musa E. M, Kamal K.T. (2015).Computational simulation of the molecular structure of benzimidazole and substituted benzimidazoles as corrosion inhibitors for brass in perchloric acid. American J. Res. Commun. 3: 143-152.

Obot I. B, Obi-Egbedi N. O, Umoren S.A. (2009). Adsorption characteristics and corrosion inhibitive properties of clotrimazole for aluminum corrosion in hydrochloric acid. Int. J. Electrochem. Sci. 4 (2009) 863-877. 